\title{
Brain, pituitary gland and nutrition
}

\section{By D. W. Lincoln, Department of Anatomy, The Medical School, University of Bristol, Bristol BS8 ITD}

The organization of a hypothalamic peptidergic neurone in terms of peptide biosynthesis and release was discussed with particular reference to the magnocellular system of neurones innervating the posterior pituitary. These neurones with their cell bodies in the paraventricular and supraoptic nuclei of the hypothalamus produce the peptides, oxytocin and vasopressin. Four principles of organization were formulated.

Oxytocin and vasopressin are produced by enzymic cleavage of two similar precursor peptides of about 19000 molecular weight during axonal transport from the hypothalamus. Thus the active hormone released from the posterior pituitary represents only $5 \%$ of the precursor from which it was formed. The remaining peptide including the neurophysin component has no known function, but other parts of the peptide precursor could be active at other sites within the nervous system where oxytocin and vasopressin have been identified by immunohistochemistry. Thus, the cleavage of a precursor peptide is the key to the production of biologically active subunits, the localization of a peptide by immunohistochemistry provides no proof of biological activity at the site in question and differential cleavage of the same precursor can result in the production of families of related peptides.

Oxytocin is released in discrete pulses. During nursing, the lactating rat releases a pulse of oxytocin every 3-10 min despite the continuous attachment of the young to the nipples; each pulse is released over a period of $<_{5} \mathrm{~s}$. The mammary gland displays a maximal contraction to a pulse of about $5 \mathrm{ng}$ oxytocin. Thus the frequency with which pulses are released becomes the more important index of efficiency when individual pulses are $>_{2}$ ng oxytocin. Similarly, it is becoming increasingly apparent that other hypothalamic peptides are released in pulses and a change in pulse frequency is clearly a major determinant in the control of puberty and seasonal reproduction by LH-RH.

Oxytocin and vasopressin containing granules are released from large stores in the posterior pituitary by action potentials operating through a $\mathrm{Ca}$-dependent mechanism of exocytosis. The number of granules released by each action potential is not constant and increases by a factor of roo- to rooo-fold as the rate of firing of single neurones increases from $I$ to 50 action potentials/s. Thus, frequency facilitation is the key to the production of pulsatile peptide release and further ensures that the background activity of the system ( $0-5$ action potentials/s) does not drain the peptide stores unnecessarily.

Neuropeptides do not readily cross the blood-brain barrier, and it is questionable whether peptides in the peripheral circulation reach the brain in sufficient 
quantities to be biologically meaningful. However, oxytocin and vasopressin producing neurones cross the blood-brain barrier as they course from the hypothalamus to the posterior pituitary and peptides in the vascular circulation can therefore reach the terminals of these neurones far more readily than they can the cell body. Indeed, $\beta$-endorphin and other opioid peptides inhibit oxytocin and vasopressin release by an action within the nerve terminal, possibly by blocking the action potential mobilization of calcium ions. The blood-brain barrier therefore serves to separate the regulatory peptides of the brain and gastrointestinal tract, though the possibility exists that peptides in the blood may modulate the release of hypothalamic peptides from neurones that terminate outside the barrier, for example, in the median eminence. 